\title{
IX.
}

Arbeiten aus dem pharmakologischen Institut der deutschen Universität zu Prag.

\section{Zur Lehre von der Wirkung substituirter Fettsäuren.}

\author{
Von \\ Dr. Julius Pohl, \\ Assistenten des Instituts.
}

Bei Gelegenheit der Untersuchung einer Reihe halogensubstituirter Säuren, die unternommen wurde, um für die Frage der Abhängigkeit der physiologisehen Wirkung von der Constitution einer Verbindung neue Anhaltspunkte zu gewinnen, stiess ich.auf Stoffe von so eigenartiger Muskelwirkung, dass mir eine Mittheilung über dieselbe gerechtfertigt erscheint. Die bezüglichen Substanzen sind: die Monochlor-, Dichlor- und Monobromessigsäure. Im Nachfolgenden theile ich die beobachteten Thatsachen und das Ergebniss der weiteren experimentellen Analyse der Giftwirkang mit. Von einer Verfolgung der Schicksale der eingeführten Substanzen im thierischen Organismus wurde abgesehen, einmal weil die die charakteristische Wirkung entfaltenden Quantitäten derselben sehr gering waren, und ferner der blosse Nachweis von Spaltungs- oder Umsetzungsproducten nicht erlaubt, diese als eigentliche Ursache der Wirkung anzusehen. Verwendet wurden stets die Natronsalze der betreffenden Säuren.

I.

Während von den Salzen der Essigsäure mit alkalischer Basis eine andere Wirkung als die erst nach sehr grossen Dosen auftretende Salzwirkung nicht bekannt ist, bilden dem gegenüber die halogensubstituirten Essigsäuren eine pharmakologisch bereits wiederholt bearbeitete Gruppe. Insbesondere die Trichloressigsäure war mehrfach Gegenstand der Discussion (Liebreich, Hermann, Binz und deren Schüler). Die Bromessigsäuren wurden 1875 von E. Ste inauer ${ }^{1}$ ) auf ihre toxikologischen Qualitäten untersucht. Der genannte

1) Virchow's Archiv. 59. Bd. S. 65. 
Autor beschäftigt sich vorwiegend mit ihrer Herzwirkung und ihrem Einfluss auf das centrale Nervensystem. Die meines Erachtens interessante Componente des Vergiftungsbildes ist ihm entgangen, vielleicht deswegen, weil dieselbe nicht immer ganz typisch entwickelt erscheint. Es ist dies die Erscheinung; dass die quergestreifte Musculatur zumeist noch bei schlagendem Herzen in hochgradige Starre versetzt wird.

In der Darstellung meiner Versuche sei der Bromessigsäure die erste Stelle eingeräumt, da sich bei ihr das Vergiftungsbild am deutlichsten, und zwar schon nach ausserordentlich kleinen Dosen entwickelt. Erhält ein Frosch, gleichgültig welcher Species, 0,0005 bis $0,05 \mathrm{~g}$ monobromessigsaures Natron, so werden, bei grösserer Dosis innerhalb weniger Minuten, bei kleineren Mengen innerhalb Stunden seine Bewegungen schwerfällig, plump, dann seltener. Zieht man die Hinterbeine $a b$, so werden sie nur schwierig angezogen, schliesslich fallen sie gelähmt nieder. Das. Thier stellt jede spontane Bewegung ein, es treten fibrilläre Muskelzuckungen auf, hier und da erfolgt ein Streckstoss, die Schwimmhäute werden gespreizt, die Bauchmuseulatur erscheint, besonders in den Flanken, eingezogen, die Musculatur der Gliedmassen wird, meist nach einem besonders stark entwickelten Anfall von Muskelflimmern, steif, hart und schliesslich so straff und starr, dass man das Thier wie einen geraden Stab in die Höhe heben kann. Die Muskelcontouren treten jetzt, wie an einem anatomischen Präparat, plastisch durch die Haut hervor, der Kopf des Thieres erscheint, infolge der Starre der Rückenmusculatur nach rückwärts gebeugt. Dabei arbeitet das Herz noch fort, aber allmählich wird die Zahl seiner Sehläge geringer, seine Kräfte erlahmen und nach einiger Zeit finden wir dasselbe stillstehend, entweder in starrer Systole (bereits von Steinauer constatirt) oder in erschlafftem Zustande.

Von den zahlreichen mir vorliegenden Versuchsprotokollen sei in Kürze eins wiedergegeben, das den gewöhnlichsten Verlauf des Vergiftungsversuches zeigt.

Versuch. Grosse Temporaria.

$6 \mathrm{~h} 49 \mathrm{~m} .0,05$ Natr. monobromacet. in den Rückenlymphsack.

$6 \mathrm{~h} 50 \mathrm{~m}$. Spontane Bewegungen hören auf.

$6 \mathrm{~h} 55 \mathrm{~m}$. Das Thier verträgt dauernd die Rückenlage.

$6 \mathrm{~h} 58 \mathrm{~m}$. Das blossgelegte Herz zeigt 12 Contractionen in $10 \mathrm{Se}$ cunden. Spreizen der Schwimmhänte, Muskelflimmern.

$7 \mathrm{~h} 04 \mathrm{~m}$. Das Thier macht vergebliche Versuche, sich zu erheben. Die hinteren Extremitäten sind gestreckt, werden steif und starr.

$7 \mathrm{~h} 16 \mathrm{~m}$. Systolischer Herzstillstand. 
Es war nun die Aufgabe gegeben, die näheren Bedingungen dieser Muskelstarre kennen zu lernen, vor Allem aber galt es, ihren Ausgangspunkt festzustellen. Zuerst erhob sich die Frage: Wirkt die Monobromessigsäure central oder peripher?

Versuch. Einer R. temporaria wird die A. femoralis dextra ligirt, die Musculatur umbunden, so dass blos der N. ischiadicus Centrum und Peripherie verbindet. Der Nerv wird durch mit physiologiseher Kochsalzlösung getränktes Filterpapier vor dem Austrocknen geschützt.

$11 \mathrm{~h} 25 \mathrm{~m}$. 0,025 Natr. monobromaceticum.

$12 \mathrm{~h}-\mathrm{m}$. Streckbewegungen beider hinteren Extremitäten.

$12 \mathrm{~h} 06 \mathrm{~m}$. Links Musculatur starr, rechts die Bewegung in allen Gelenken frei.

$12 \mathrm{~h} 08 \mathrm{~m}$. Reflexbewegungen der rechten Extremität durch mechanisehe Reizung der Haut der linken auslösbar.

$12 \mathrm{~h} 10 \mathrm{~m}$. Bewegung des rechten Beines mit nachfolgender Erschlaffung.

$12 \mathrm{~h} 15 \mathrm{~m}$. Links die Starre ad maximum ausgebildet, alle Gelenke fixirt; rechts normale Verhältnisse. Rechter Ischiadicus bei $47 \mathrm{~cm}$ Rollen= abstand faradisch erregbar.

Der Versuch lehrt, wie viele ähnliche von mir angestellten, dass die Museulatur vom Salz durchströmt werden muss, um starr zu werden, dass sonach die Starre peripheren Ursprungs ist.

Zur Entscheidung der Frage, welcher Theil des peripheren Nervmuskelapparates zumeist rom Gifte betroffen wird, wiederholte ich den Versuch am curarisirten Thier. Es trat an demselben keine Starre auf. Schon glaubte ich, in der genannten Säure ein Gift gefunden zu haben; das in dem Sinne, wie das Muscarin auf die Nervenendigungen der glatten Muskeln, eine erregende Wirkung auf die Nervenendigungen der quergestreiften Musculatur ausübt und das sonach als typischer Antagonist des Curare anzusehen wäre, als mich weitere Versuche belehrten, dass der Sachverhalt ein complicirterer ist. Durchtrennt man den N. ischiadicus eines Frosches vor Application der Monobromessigsäure, so bleibt das betreffende Bein, bei vollständig entwickelter Starre der übrigen Musculatur, normal, seine Musculatur weich, elastisch, das Fussgelenk frei beweglich. Obwohl also Muskeln wie Nervenendigungen vom Gifte umspült werden, tritt die Starre dennoch nicht auf. Es lag nahe, anzunehmen, dass das Zuströmen der normalen, continuirlich durch die Nervenbahn zu geleiteten Impulse eine Bedingung der Starre ist, und zwar konnte man sich vorstellen, dass es sich um eine Erregbarkeitsänderung der Muskelfaser handelt, derart, dass die Musculatur auf normale Reize in abnorm intensiver Weise - mit Danercontraction - reagirt, dass sich aber die gesteigerte Irritabilität nicht äussert, wenn die aus- 
lösenden Impulse fehlen. In der That zeigte es sich, dass es möglich ist, wenn man die normalen, durch den Nerven zufliessenden Reize durch den elektrischen Strom ersetzt, auch an einer Extremität, die infolge der Rückenmarks- oder Ischiadicusdurchschneidung von der Starre frei blieb, nachträglich -Starre zu erregen. Man braucht blos den durchschnittenen Ischiadicus durch $1 / 2-1$ Minute mit schwachen Strömen zu faradisiren, so tritt meist sofort oder doch innerhalb der nächsten Minuten die Starre - leicht kenntlich an der Steifigkeit des Fussgelenkes - in Erscheinung. Es ist selbstverständlich, dass in vielfachen Controlversuchen erhärtet wurde, dass es am normalen Thiere oder der von der Circulation ausgeschalteten Extremität eines mit Monobromessigsäure vergifteten Thieres niemals gelingt, durch gleich schwache Ströme einen der Starre ähnlichen Zustand herbeizuführen.

Durch die letzteren Versuche war das Ausbleiben der Starre am curarisirten Thier aufgeklärt: die Lähmung der Nervenendigungen hatte das Uebertragen der physiologischen Reize vom Nerv zum Muskel unmöglich gemacht. Dieser Auffassung entsprechend gelingt es, an einem mit Curare und Monobromessigsäure vergifteten Thier durch directe Muskelreizung die Starre zu erzwingen.

Versuch. Mittelgrosse R. temporaria.

$4 \mathrm{~h}-\mathrm{m}$. Einige Tropfen einer Curarelösung in den Rückenlymphsa.ck.

$4 \mathrm{~h} 24 \mathrm{~m}$. Reflexerregbarkeit vollständig aufgehoben. 0,025 Natrium monobromaceticum.

$5 \mathrm{~h} 20 \mathrm{~m}$. Der Zustand des Thieres unverändert, der blossgelegte rechte Ischiadicus durch die stärksten Ströme nicht mehr erregbar. Ueberstreichen des Gastrocnemius mit den Elektroden macht denselben in Kurzem starr, das Knie und Fussgelenk steif und unbeweglich.

$7 \mathrm{~h}-\mathrm{m}$. Rechts Starre, linke Extremität wie die übrige Körpermusculatur frei.

Da also Lähmung der Nervenendapparate durch Curare das Anftreten der Starre nicht unmöglich macht, so wird man zu dem Schlusse gedrängt, dass die durch das Gift gesetzte Muskelveränderung auch in dem Falle vorbanden ist, wo die Starre wegen Mangel äusseren Reizes nicht auftritt. An einem so veränderten Muskel treten die chemischen und molecularen Vorgänge, als deren Ietztes sinnfälliges Product die Contraction in Erscheinung tritt, auf Einwirkung physiologischer Reize zwar in normaler Weise ein, sind sie aber einmal ausgelöst, so spielen sie sich in abnormer Intensität und Qualität ab, infolge dessen der Muskel seine Fähigkeit, za erschlaffen, verliert. Durch Versuche mit einem Apparat, weleher die Muskelcontrac- 
tion verzeichnet, lässt sich diese Veränderung des Muskelzustandes leicht graphisch zum Ausdruck bringen. Man sieht an solehen Curven, dass jedesmal nach der Contraction die Erschlaffung eine minder vollständige wird und schliesslich ganz ausbleibt. Dieses Verhalten erinnert äusserlich, wenngleich nur entfernt, an jenes des. Veratrinmuskels. Beim Veratrinmuskel tritt jedoch die Erschlaffung immer wieder, wenn auch nach ungewöhnlich langer Contractionsdauer, ein und ist schliesslich eine vollständige. Beim Monobromessigsäuremuskel erfolgt die Erschlaffung sofort nach Auf hören der Reizzufuhr, ist aber nur unvollständig. Der Eintritt der Dauerverkürzung kann durch Anwendung stärkerer elektrischer Reize beschleunigt werden; sie tritt jedoch auch nach Anwendung schwächster elektrischer Ströme ein, und zwar, wenn dieselben durch Pausen unterbrochen werden, etwa ebenso rasch, als wenn man dieselben die ganze Zeit über einwirken lässt. Man hat daher den Eindruck, dass hier, im Gegenșatz zum Veratrin, die Veränderung der Muskelsubstanz, welche die Dauercontraction bewirkt, sich nur langsam unter dem Einfluss des elektrischen Stroms vollzieht, ähnlich wie beim einfachen Vergiftungsversuch die Starre nicht mit einem Schlag im Gefolge einer kräftigen Bewegung auftritt, sondern successive in fast regelmässiger Steigerung sich entwickelt. Eine Analogie für die eigenthümliche Erscheinung, dass die hochgradige Erregbarkeitsveränderung der Muskelsubstanz nur nach Binzutritt von vitalen oder elektrisehen Reizen wahrnehmbar wird, kann man in dem bekannten Verhalten des reflexvermittelnden Apparates des Rïckenmarkes unter dem Einfluss kleiner Strychningaben finden. Hier kann Steigerung der Reflexerregbarkeit im höchsten Grade vorbanden sein, obne dass Tetanus auftritt, falls jeder reflexauslösende Reiz ferngehalten wird.

Die Aehnlichkeit, welche die Wirkung der Monobromessigsäure auf die quergestreifte Musculatur nach anfänglichen Versuchen mit jener des Muscarins auf die glatte zu haben schien, legte es nahe, beim Muscarin den Einfluss äusserer Reize auf 'das Zustandekommen der Myose za untersuchen. In der That tritt dieselbe entschieden rascher und intensiver auf, wenn das Auge nach der Instillation dem vollen Tageslicht ausgesetzt wird, als wenn man es im Dunkeln hält. Bei bezüglichen Kaninchenversuchen zeigte sich oft eine Differenz von rund $3-4 \mathrm{~mm}$ in der Pupillenweite zu Gunsten des belichteten Auges, eine Differenz, die sich nach gleichmässiger Belichtung der Augen allmählich ausglich. Doch begründet dieses Verhalten im Hinblick auf den oben geführten Nachweis, dass die Monobromessigsäure nicht die Nervenendigungen, sondern den Muskel selbst ver- 
ändert, eine nur ganz oberflächliche Analogie zwischen Monobromessigsäure und Mascarin. Es dürfte dasselbe doch insofern von Interesse sein, als es die Inconstanz der Muscarinwirkung beim Menschen wenigstens zum Theil erklären könnte.

Während beim Kaltblüter die durch die Monobromessigsäure veranlasste Ausschaltung der quergestreiften Musculatur das Fortbestehen des Lebens nicht unmöglich macht, muss sich beim Warmblüter an die Starre, speciell der Athmungsmuskeln, der Erstickungstod anschliessen. Bei Kaninchen erfolgen auf eine Dosis von $0,07 \mathrm{~g}$ pro Kilo (intravenös gegeben) nach kurz dauerndem fibrillären Zucken der Musculatur äusserst heftige, rasch in Starre übergehende Convulsionen, worauf in wenigen Secunden der Tod eintritt. Kymographionversuche am curarisirten Thier, bei dem die Starre ausbleibt, zeigen, dass der peripheren Muskelwirkung eine Lähmung des Vasomotorencentrums parallel geht, ein Umstand, der den Erstickungstod nur noch beschleunigt.

Es sei hier erwähnt, dass die wenigen mitgetheilten Ver"suchsprotokolle nur den durchschnittlichen Typus des Vergiftungsbildes darstellen, dass sich in einzelnen von Hunderten angestellter Experimente öfters Unregelmässigkeiten seines Ablaufes einstellten. Speciell zeigte sich die Herzwirkung, der von Anfang an besondere Aufmerksamkeit geschenkt wurde, von unerwünschter Unregelmässigkeit. Starke systolische, selbst intensive Starre des Herzmuskels bietende Herzstillstände wechselten mit diastolischen Stillständen, ohne dass es mir gelungen ist, die Ursache dieser Verschiedenheiten zu ergründen. Bei mit dem Willia m s'schen Froschberzapparat angestellten Versuchen konnte in keinem Falle eine Erhöhung der Leistungsfähigkeit des Herzens nachgewiesen werden.

Andere Stoffe, von denen bekannt ist, dass sie Muskelstarre hervorrufen (Chloroform, Aether, Säuren, Coffein und die Xanthinderivate), haben in ihrer Muskelwirkung mit der Monobromessigsäure nur äusserlich.Aehnlichkeit, da sich dieselbe auch am curarisirten Thier einstellt.

Ich muss weiter noch hervorheben, dass eine Säuerung des Muskels in der Starre bei Prüfung gegen Lackmus nicht nachweisbar war.

\section{II.}

Nach meinen Versuchen über die toxische Wirkung der mit Chlor substituirten Essigsäuren - Monochlor- und Dichloressigsäure - zeigt sich diesen die Fähigkeit gemein, neben Narkose noch Muskelstarre, allerdings nicht ganz regelmässig, hervorzurufen. Die Tr̈ichloressig- 
säure führt erst nach grossen Gaben $(0,3 \mathrm{~g})$ centrale motorische Parese, stundenlang andanernde Narkose schliesslicl den Tod durch diastolischen Herzstillstand herbei. Eine Muskelwirkung fehlt. Auch die Monojodessigsäure liess bemerkenswerthe Vergiftungssymptome vermissen.

Versuch. Eine mittelgrosse R. temporaria erhält

$5 \mathrm{~h} 35 . \mathrm{m} 0,1 \mathrm{~g}$ monochloressigsanres Natron.

$5 \mathrm{~h} 58 \mathrm{~m}$. Das Thier hält die vorderen Extremitäten fest an den Leib gezogen, so dass es bei den allmählich seltener und schwächer ausfallenden Sprungbewegungen auf den Bauch fällt. Hebt man die hinteren Extremitäten vom Körper ab, so werden sie nicht angezogen, fallen schlaff nieder.

6 h $25 \mathrm{~m}$. Das Thier bleibt dauernd in der Rückenlage. Tetanische Streckung der hinteren Extremität. Muskelcontraction vom Nerven aus bei $260 \mathrm{~mm}$, direct bei $125 \mathrm{~mm}$ Rollenabstand zu erzielen. Während der elektrischen Untersuchung fibrilläre Zuckungen in den Adductoren. Die Musculatur der Extremitäten wird steif und starr. Das blossgelegte Herz systolisch contrahirt.

Versuch. Grosse Esculenta.

Das Thier, das an 2 Tagen je $0,05 \mathrm{~g}$ Natr. dichloraceticum injicirt bekommen latte, ohne dass Vergiftungserscheinungen aufgetreten waren, erhält am dritten Tage um

$10 \mathrm{~h}-\mathrm{m} \quad 0,1$ Natr. dichloraceticum.

$10 \mathrm{~h} 11 \mathrm{~m}$. Fibrilläre Zuckungen, dann tetanische Streckung der Extremitäten. Muskeln bleiben im Anschluiss an eine solche Streckung starr, Gelenke steif. Allmählich wird die gesammte Musculatur starr. Die blossgelegten Muskeln reagiren gegen empfindliches Lackmuspapier alkalisch. Herz schlägt verlangsamt.

$11 \mathrm{~h} \mathrm{-} \mathrm{m.} \mathrm{Herzstillstand} \mathrm{in} \mathrm{schlaffem} \mathrm{Zustande.}$

In anderen Fällen blieb die Starre aus. So trat bei einer R. temporaria nach 0,3 Natr. dichloraceticum Hypnose, eine an Veratrinwirkung èrinnernde Verlangsamung der Muskelerschlaffung, schliesslich Tod unter diastolischem Herzstillstand, aber keine Starre ein. Die Inconstanz dieser Muskelwirkung, die, selbst ausgebildet, zu keinen neuen pharmakologischen Gesichtspunkten fuhrte, liess eingehendere Warmblüterversuche wenig aussichtsvoll erscheinen. Es sei nur kurz über einen Kymographionversuch an einem $2170 \mathrm{~g}$ schweren Kaninchen berichtet, das noch nach $0,7 \mathrm{~g}$ intravenös eingeführtem monochloressigsaurem Natrium keine Erscheinungen von Seiten des Muskel-, Circulations- und Respirationsapparates darbot. Erst als die injicirte Menge $1 \mathrm{~g}$ überstieg, trat Unregelmässigkeit der Respiration, Zucken der Extremitäten, schliesslich Absinken des Blutdrucks auf Null, bedingt durch Lähmung des Vasomotorencentrums, auf. 
III.

Wie in Vorstehendem entwickelt wurde; sind die Monobrom-, Monochlor- und Dichloressigsäure in absteigendem Maasse befähigt, Muskelstarre herbeizuführen. Es hat also das in ähnlicher Richtung unwirksame Essigsäuremolekül dureh Vertretung eines oder mehrerer Wasserstoffatome durch die Halogene physiologische Eigensehaften erhalten, die ihm früher mangelten und die auch dem Substituenten abgehen. Freies Chlor, Brom, Jod, unterbromigsaure und bromsaure Salze entfalten keine analoge Muskelwirkung. In dieser Beeinflussung des physiologischen Verhaltens durch Aenderung der Constitution war die Aufforderung gegeben, noch andere substituirte Säuren pharmakologisch zu untersuchen. Die Vergiftungen mit diesen lieferten aber zumeist so gleichförmige Bilder, dass es genügt, die an Fröschen hierïber gewonnenen Erfahrungen tabellarisch zusammenzustellen.

\begin{tabular}{|c|c|c|}
\hline Substanz & $\begin{array}{l}\text { Dosis } \\
\text { in } g\end{array}$ & Vergiftungssymptome \\
\hline Cyanessigsüure . . . . . & 0,1 & Unwirksam. \\
\hline Cyanessigsäure ...... & 0,2 & $\begin{array}{l}\text { Lang andauernde Narkose, diastolischer Herz- } \\
\text { stillstand. }\end{array}$ \\
\hline Monochlorpropionsäure & 0,2 & Hypnose. \\
\hline Monojodpropionsäure . . & 0,2 & $\begin{array}{l}\text { Anfangs gesteigerte Reflexerregbarkeit, dann voll- } \\
\text { ständiger Verlust derselben, Narkose. }\end{array}$ \\
\hline Crotonsäure ..... & 0,05 & Hypnose. \\
\hline Monochlorerotonsäure. . & $0,1-0,15$ & $\begin{array}{l}\text { Fibrilläre Muskelzuckungen, Narkose, diasto- } \\
\text { liseber Herzstillstand. }\end{array}$ \\
\hline Trichlormilchsäure ... & 0,2 & Ohne Wirkung. \\
\hline Monobrombernsteinsäure & 0,1 & Hypnose, diastolischer Herzstillstand. \\
\hline Bibrombernsteinsänre. . & 0,2 & Hypnose. \\
\hline $\begin{array}{l}\text { Pyruvinsäure . . . . . } \\
\quad\left(\mathrm{CH}_{3} . \mathrm{CO} \mathrm{COOH}\right)\end{array}$ & 一 & Ohne Wirkung. \\
\hline Bibrompyruvinsäure. . . & 0,02 & $\begin{array}{l}\text { Aussetzen der Respiration, Pulsverlangsamung, } \\
\text { zumeist systolischer Herzstillstand; tödtet oft } \\
\text { binnen } 10 \text { Minuten. }\end{array}$ \\
\hline
\end{tabular}

Wie ersichtlich, stellte sich am häufigsten eine narkotische Wirkung ein. Wie sehr man jedoch fehlginge, den Halogengehalt als Ursache und Maassstab der schlafmachenden Wirkung anzusehen, zeigt der Vergleich der Wirkung des krotonsauren und monochlorcrotonsauren Natrons, von denen das nicht substituirte bereits in der um die Hälfte kleineren Dosis, als das substituirte, narkotisch wirkt. Aehnliches beobachtete Heinrich May e ${ }^{1}{ }^{1}$ ) bezïglich des buttersauren und trichlorbuttersauren Natrons. Die Cyanessigsäure erwies sich, gleich manchen anderen bekannten Cyanverbindungen, als relativ

1) Dieses Archiv. XXI. Bd. S. 97 u. 119. 
150 IX. Poнг, Zur Lehre von der Wirkung substituirter Fettsăuren.

unschädlich, was darauf hindeutet, dass sie im Froschorganismus keine Spaltung erfährt.

Diese Versuche lehren neuerdings, dass Betrachtung der Constitutionsformel an sich nicht direct Aufschliusse über die Wirkung eines Stoffes gestattet, dass letztere vielmehr auch von Eigenschaften abhängig sein muss, welche, wie die Zersetzlichkeit, Löslichkeit und molecularchemisches Verhalten, in der empirischen, wie in der Structurformel keinen oder nur ungenügenden Ausdruck finden. 